\title{
Ambulatory scintigraphic assessment of transient changes in left ventricular function: a new method for detection of silent myocardial ischaemia
}

\author{
M. Pfisterer, S. Regenass, J. Müller-Brand and F. Burkart \\ Divisions of Cardiology and Nuclear Medicine, University Hospital, Basel, Switzerland
}

KEY WORDS: Silent ischaemia, left ventricular function, ambulatory monitoring, radionuclide angiocardiography.

\begin{abstract}
Demonstration of ischaemic left ventricular dysfunction in the absence of chest pain should provide important confirmation of silent myocardial ischaemia in patients with asymptomatic ST segment changes. For this purpose, a new portable scintillation probe (VEST) similar to a miniaturized nuclear stethoscope combined with a Holter ECG was evaluated. After standard equilibrium radionuclide angiocardiography with technetium-99m labelled red blood cells, the VEST was postioned under gamma-camera control and data were recorded from $1-12 \mathrm{~h}$ in 61 unselected patients. Ejection fraction ( $L V E F)$, relative changes in volumes, heart rate and ST segment changes were determined. Reproducibility of $L V E F$ at rest $(r=0.91$; variability $3.8 \pm 3 \%, N=19)$ and during exercise $(r=0.98$; variability $3.2 \pm 2 \%, N=19)$ was good. In 15 asymptomatic exercise tests four different patterns of LVEF and ST segment responses were identified: (I) decrease in LVEF followed by significant $S T$ depression (five times); (2) ST depression followed by decrease in LVEF (three times); (3) decrease in LVEF without significant ST changes (three times); and (4) ST depression without significant LVEF change (four times). In this still small series, patterns (I) to (3) corresponded to patients with documented coronary artery disease, which was not the case for pattern (4). For detection of silent ischaemia at rest, a decrease in LVEF of $>5 \%$ lasting for $>1$ min was defined as ischaemic $L V$ dysfunction. Using this definition, four spontaneous episodes of silent $L V$ dysfunction could be demonstrated in two of three CCU patients with unstable angina during 160-680 min of data recordings without simultaneous ST changes. Based on this initial experience, we conclude that VEST is a reproducible method to detect transient global LV dysfunction and will be useful to confirm silent ischaemia in otherwise uncertain ST segment changes.
\end{abstract}

\section{Introduction}

The diagnosis of silent ischaemia is limited by the lack of a gold standard for detection of myocardial ischaemia. Whereas there are invasive haemodynamic, metabolic and angiographic as well as noninvasive scintigraphic methods to confirm asymptomatic ST segment depression as a sign of myocardial ischaemia, these methods are restricted mostly to use in the laboratory. Tests to provoke (silent) myocardial ischaemia include mental stress, a cold pressure test and drug administration such as ergonovine maleate or dipyridamole besides physical exercise testing. However, spontaneous episodes of silent myocardial ischaemia rarely occur under laboratory conditions. Therefore, the only method for detecting such transient ischaemic episodes which has gained widespread application is ambulatory Holter ECG monitoring ${ }^{[1]}$. Critical analysis of ST segment of Cardiology, University Hospital, $\mathrm{CH}-4031$ Basel, Switzerland. changes during daily activities showed that despite high quality recorders and stringent criteria for diagnosis of silent ischaemia, there remains a fairly high percentage of false or uncertain positive (and negative) results ${ }^{[2]}$. These findings were confirmed by probability calculations indicating that even socalled significant ST segment depression in an asymptomatic patient with low probability of disease may not just be labelled 'silent ischaemia' but require confirmation by another method ${ }^{[3]}$.

With the introduction of a new portable scintillation probe (VEST) ${ }^{[4.5]}$, similar to a miniaturized nuclear stethoscope ${ }^{[6]}$ combined with a Holter ECG recording system, there is the ability to detect transient left ventricular dysfunction during ischaemic episodes on an ambulatory basis. Demonstration of ischaemic left ventricular dysfunction in the absence of chest pain should provide important confirmation of silent myocardial ischaemia in patients with asymptomatic ST segment changes. The aim of this report is to describe and validate the 'VEST' method 
in view of this purpose and to present initial findings in patients with silent myocardial ischaemia.

\section{Patients and methods}

\section{PATIENTS}

To test and validate the VEST system, 61 unselected patients referred for diagnostic radionuclide angiocardiography were asked to participate in the protocol. The total patient population has been previously described in more detail ${ }^{[7]}$. Out of 98 exercise tests performed in these patients, 15 were totally asymptomatic without anti-ischaemic drugs but showed either significant ST depression and/or a significant drop in left ventricular ejection fraction (LVEF) during exercise. The presence or absence of coronary artery disease was confirmed by coronary angiography or a history/follow-up of myocardial infarction. Three additional patients with unstable angina were studied in the coronary care unit at rest over 160 to $680 \mathrm{~min}$.

\section{RADIONUCLIDE ANGIOCARDIOGRAPHY/VEST}

Standard radionuclide angiocardiography was performed at rest and if required during exercise after in vivo labelling of red blood cells with $25 \mathrm{mCi}$ of technetium-99m as previously reported and validated $^{[8]}$. After this diagnostic test, the detector of the VEST system was positioned over the left ventricle under gamma camera control in a left anterior oblique projection such that the left atrium and the right ventricle were not covered by it. The VEST detector consisted of a $5-\mathrm{cm}$ diameter sodium iodide crystal with a parallel hole collimator to maximize sensitivity and field uniformity. To hold the detector in place over the left ventricle and lung, it was fitted with a firm plastic vest-like garment. Counts detected by the crystal and signals from a chest lead of the ECG were recorded on a modified Holter ECGrecorder as previously described ${ }^{[7]}$.

Data analysis consisted of reviewing radionuclide data for technical adequacy and to rule out major motion artefacts as indicated by a sudden shift in total counts. Thereafter, ECG and radionuclide data
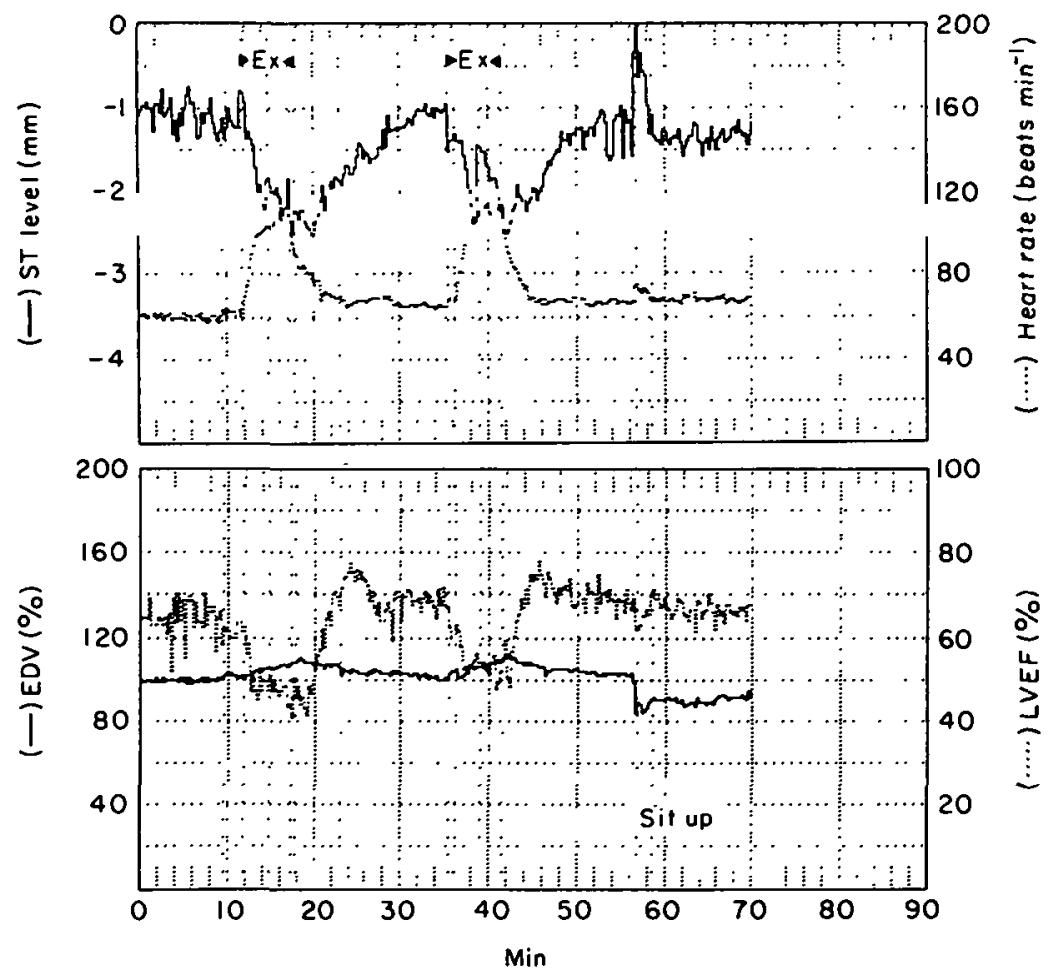

Figure I Silent ischaemia during exercise (H.Z., 55 years, two-vessel CAD). Note decrease in LVEF during exercise before significant ST depression. Note also the overshoot increase in LVEF after exercise and the artefact after $57 \mathrm{~min}$ when the patient sat up: sudden drop in total end-diastolic volume. ex, Exercise. 
were summed for 15-s intervals to determine ejection fraction, relative changes in end-diastolic and endsystolic volumes, heart rate, cardiac output and ST segment changes. On the basis of previous studies $^{[5.7]}$, a fixed background correction of $75 \%$ of end-diastolic counts was used to calculate LVEF values. Data were displayed graphically and numerically for analysis. Based on results of variability measurements, a decrease of $>5 \%$ was defined as 'ischaemic' left ventricular dysfunction. For the ST segment, a depression of $>1 \mathrm{~mm}$ was assumed to be significant.

All exercise tests were performed on a supine bicycle ergometer up to symptom limitation (chest pain, dyspnea or fatigue). For determination of reproducibility of obtained results, some patients had two exercise tests according to previously described protocols $^{[7]}$.

\section{RESULTS}

Reproducibility of LVEF determined by the VEST system at rest $(r=0.93 ; n=19)$ and during exercise $(r=0.96 ; n=19)$ was good with a low variability of repeated measurements (at rest: $\pm 3 \%$, during exercise: $\pm 2 \%$ ). Therefore, the exercise induced change in LVEF (delta EF\% rest - exercise) was also good $(r=0.88)$, indicating that transient changes in LVEF can be consistently determined by this method.

In the 15 asymptomatic exercise tests, four different patterns of LVEF and ST segment responses were identified: (1) decrease in LVEF followed by a significant ST depression (five times); (2) ST depression followed by a decrease in LVEF (three times); (3) decrease in LVEF without significant ST changes (three times); and (4) ST depression without significant LVEF change (four times). Examples of each of these are shown in Figs 1-4. In this still small series, patterns (1) to (3) corresponded to patients with documented coronary artery disease, which was not the case for pattern (4). For detection of silent ischaemia at rest, a decrease in LVEF of $>5 \%$ lasting for $>1 \mathrm{~min}$ was defined as ischaemic left ventricular dysfunction. Using this definition four spontaneous episodes of silent left ventricular dysfunction could be demonstrated at rest in two of three coronary care unit patients with unstable angina during 160-680 min of data recording without simultaneous ST

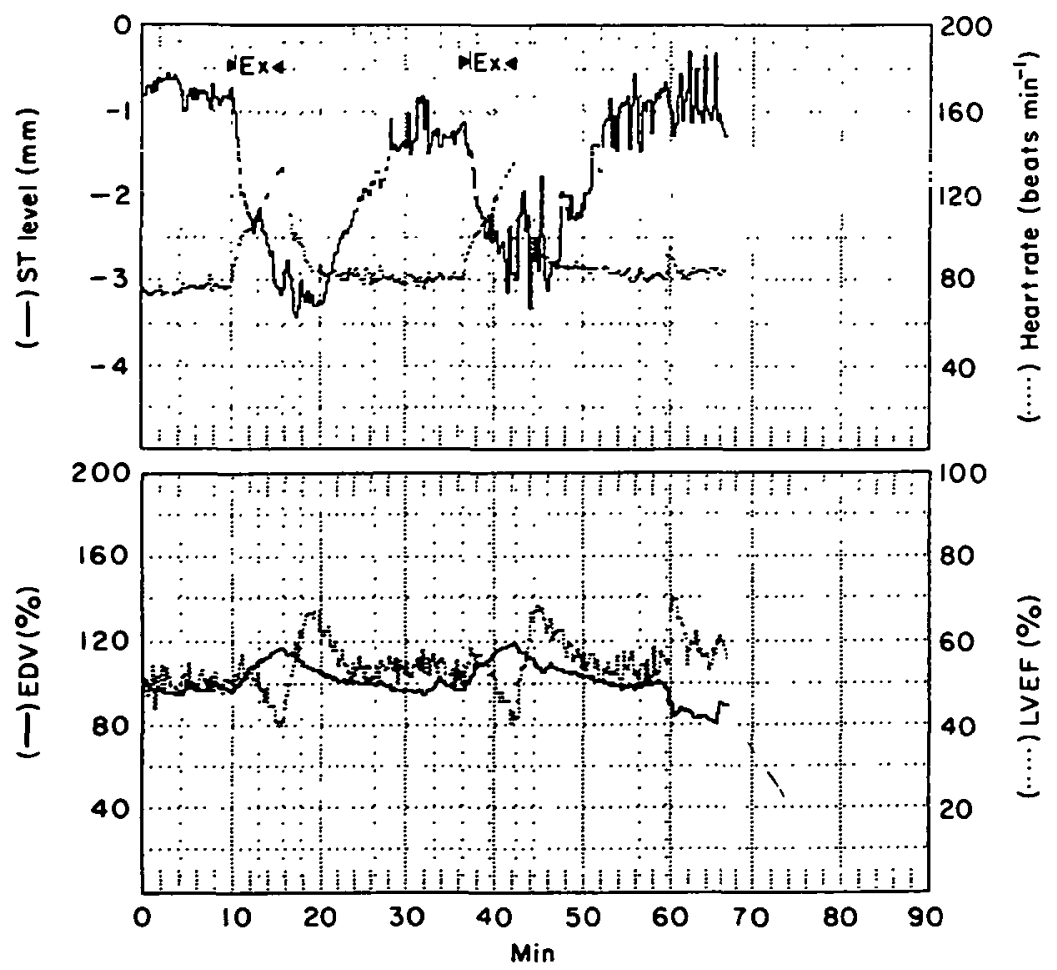

Figure 2 Silent ischaemia during exercise (T.S., 57 years, two-vessel CAD). Note decrease in LVEF only during second level of exercise after significant ST depression. Note again the overshoot increase in LVEF after exercise. 


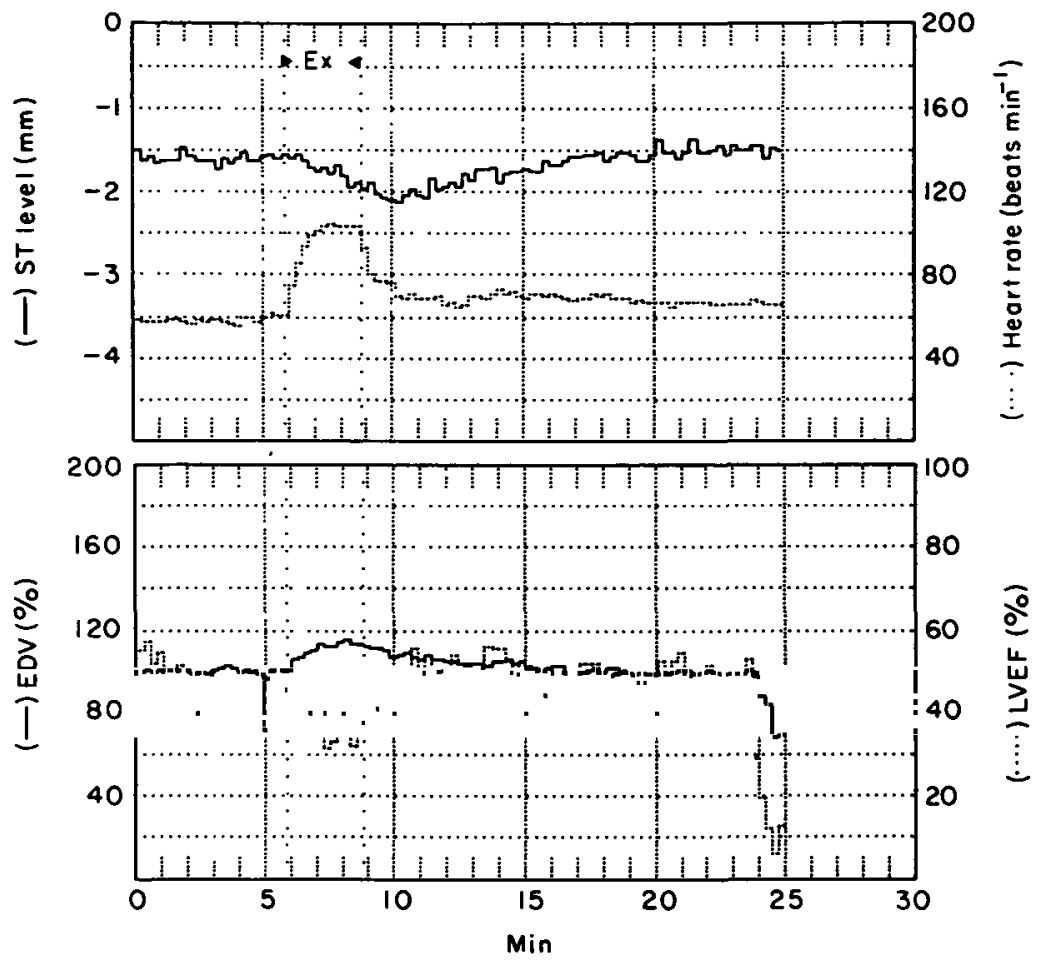

Figure 3 Silent ischaemia during exercise (R.W., 66 years, previous non-Q anterior myocardial infarction). Note significant fall in LVEF during exercise without $>1 \mathrm{~mm} \mathrm{ST} \mathrm{de-}$ pression which occurs mainly after exercise.

changes. An example is shown in Fig. 5. This patient had anginal chest pain while walking from the gamma camera to his bed, where pain even increased without significant ST depression at this point in time but with marked depression of LVEF. Furtheron, he had two episodes of prolonged $(6 \mathrm{~min}$ and $20 \mathrm{~min}$ ) depression of LVEF of $>5 \%$ without chest pain or ST depression.

\section{Discussion}

Demonstration of ischaemic left ventricular dysfunction in the absence of chest pain can provide important confirmation of silent myocardial ischaemia in patients with asymptomatic ST segment depression. This study demonstrates that using the new portable VEST system, transient changes in radionuclide LVEF can be determined reproducibly. Based on the initial experience of our laboratory, four different patterns of LVEF and ST segment changes could be identified and it was shown that even spontaneous episodes of silent ischaemic left ventricular dysfunction can be registered.

On the basis of experimental studies, characteristic changes of ischaemic events have been identified ${ }^{[9,10]}$ : if myocardial perfusion becomes insufficient, changes in metabolism are first detected, followed by diastolic and then systolic functional impairment; electrocardiographic changes occur only at a later phase, but still before appearance of chest pain. This chain of events may explain observed patterns (1) and (3) in this study where functional ischaemic changes appeared before or without significant ST segment shifts. For pattern (2), however, other factors have to be discussed: with a nuclear probe as used in the VEST system, only changes in global LVEF may be detected. In a previous study we could show that hypoperfusion even of a large area as the anterior wall does not have to induce a significant $5 \%$ fall in LVEF if the other parts of the myocardium are normally perfused and behaving ${ }^{[11]}$. Since ischaemic heart disease is a regional 


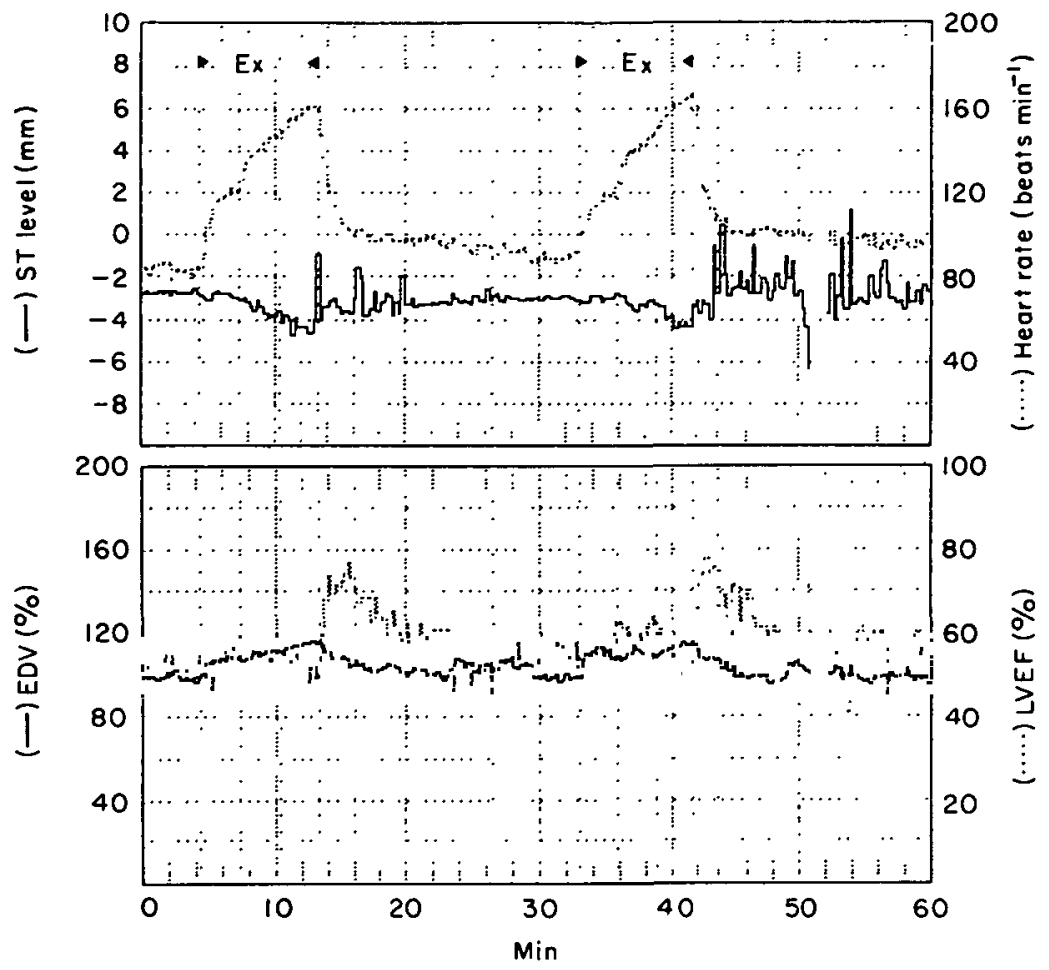

Figure 4 Silent ischaemia during exercise? (H.R., 52 years, normal coronaries). Note there is no significant change in LVEF during exercise despite a $>1 \mathrm{~mm}$ ST depression. Note also again the marked overshoot increase in LVEF after exercise.

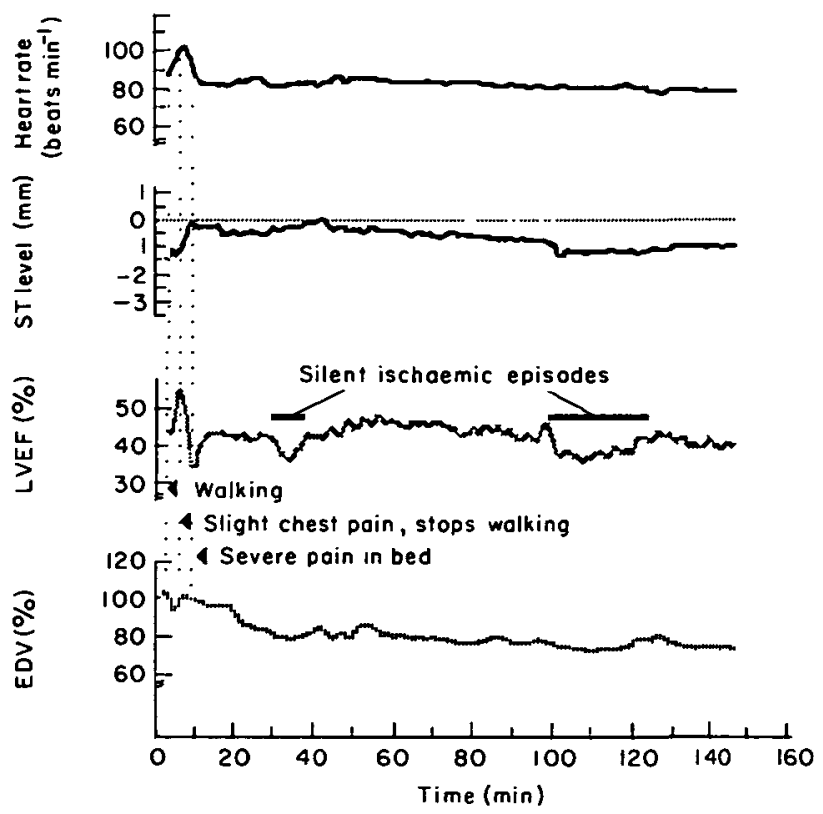

Figure 5 Silent ischaemia at rest (A.H., 70 years, unstable angina, three-vessel $\mathrm{CAD}$ ). Note symptomatic and prolonged silent ischaemic episodes as detected by long-term LVEF monitoring (cf. text). 
disease of the myocardium, there may be non-uniformity of contraction which may explain delayed impairment of global function as observed in pattern (2). Furthermore, this may or may not be detected by electrocardiographic changes depending on the leads registered. Finally, pattern (4), excludes ischaemı with some degree of certainty only if there is a normal increase in LVEF of $>5 \%$ during exercise as in diagnostic radionuclide studies. These considerations, lead to the conclusion that there are some limitations inherent to radionuclide probe measurements as presented here: (1) only global function and relative haemodynamic changes can be observed and (2) functional changes of the apical area of the left ventricle may be weighted more than basal changes due to the detector position. In addition, probe displacement during ambulatory recording remains an important problem.

On the other hand, Kayden and co-workers have recently demonstrated that transient ischaemic left ventricular dysfunction detected by the VEST system is clinically relevant ${ }^{[12]}$ : in a prospective study, they reported that this finding was more sensitive than symptoms or electrocardiographic exercise testing to identify patients at risk for future ischaemic events after acute myocardial infarction and thrombolysis. On the basis of their and our initial experience, we conclude that VEST is a reproducible method of detecting transient global left ventricular dysfunction which will be useful to confirm silent ischaemia in otherwise uncertain ST segment changes and may have important prognostic power.

\section{References}

[1] Selwyn AP The value of Holter monitoring in managing patients with coronary artery disease. Circulation 1987; 75/11: 31 .
[2] Deanfield JE, Shea M. Ribiero P et al. Transient STsegment depression as a marker of myocardial ischemia during daily life. Am J Cardiol 1984: 54: 1195.

[3] Rozanski A, Berman DS. Silent myocardial sschemia. Pathophysiology, frequency of occurrence and approaches toward detection. Am Heart J 1987; 114: 615.

[4] Wilson RA. Sullivan PJ. Moore RH et al. An ambulatory ventricular function monitor: validation and preliminary results. Am J Cardiol 1983: 52: 601 .

[5] Tamaki N, Gill JB, Moore RH et al. Cardiac response to daily actıvities and exercise in normal subjects assessed by an ambulatory function monitor. Am J Cardiol 1987; 59: 1164.

[6] Wagner HN, Wake R, Nickoloff E, Natarajan TK. The nuclear stethoscope: a simple device for the generation of left ventricular volume curves. Am J Cardiol 1976; $23: 747$.

[7] Regenass S, Pfisterer M, Bassignana V, Müller-Brand J. Erfassung passagerer LV-Dysfunktion mittels ambulantem nuklearmedizinıschem Monitorıng: Evaluation einer neuer Method. Thesis. University of Basel Medical School (in press).

[8] Pfisterer M, Ricci DR, Schuler G, Swanson SS, Gordon DG, Peterson K, Ashburn WL. Validity of left ventricular ejection fractions measured at rest and peak exercise by equilibrium radionuclide angiography using short acquisition times. J Nucl Med 1979; 20: 484.

[9] Upton MT, Rerych SK, Newman GE et al. Detecting abnormalities in left ventricular function during exercise before angina and ST-segment depression. Circulation 1980; 67: 341 .

[10] Sigwart U, Grbic M Rôle de la pression coronarienne sur la relaxation du ventricule gauche. Schweiz med Wschr 1984; 114: 1618.

[11] Pfisterer M, Glaus L, Burkart F. Comparative effects of nitroglycerin, nifedipine and metoprolol on regiona! left ventricular function in patients with one-vessel coronary disease. Circulation 1983; 67: 291

[12] Kayden DS, Wackers FJ, Francis CK, Zaret BL. Silent left ventricular dysfunction during ambulatory radionuclide monitoring following thrombolysis: a potentıal predictor of subsequent cardiac morbidity. Am J. Cardiol 1988; in press. 